\title{
Two Dimensional Micro Magnetic Sensor Array
}

$\begin{array}{lll}\text { Member } & \text { Desheng LI } & \text { (The University of Tokyo) } \\ \text { Member } & \text { Kyoseok CHUN } & \text { (The University of Tokyo) } \\ \text { Member } & \text { Tetsuhiko IIZUKA } & \text { (The University of Tokyo) } \\ \text { Member } & \text { Hiroyuki FUJITA } & \text { (The University of Tokyo) }\end{array}$

Summary

This paper proposes two new kinds of two dimensional micro magnetic sensor arrays, which are designed for a position sensor in a micro superconducting magnetic conveyance system with feedback control. One is a magnetic switch array with a nickel cantilever structure fabricated by electro-plating. Another one is a Hall sensor array consisted of so-called a MOS-Hall element, which is a MOS device in fact, but works as a Hall element. We have fabricated these two different types of sensor arrays by micromachining process, and tested their performances. The sensing principle, fabrication process and experimental results are reported.

Key words: Micromachine, Magnetic sensor, Switch, Hall element, Magnetic conveyance

\section{INTRODUCTION}

Recent years, the studies on micro conveyance system have made a large progress, and several micro conveyance systems have been reported [1] [3]. In the present study, the authors are aiming at realizing a micro conveyance system with feedback control based on superconducting magnetic levitation (see Figure 1) [4], which is expected to be useful as a transportation system for advanced semiconductor fabrication apparatus. In order to obtain precise position control of conveyor in the system, it is necessary to develop a two dimensional position sensor to detect its position. We assume that one or several permanent magnets are attached to the slider. Hence, it is possible to detect its position by sensing the presence of the attached magnet. For this purpose, authors developed two different types of two dimensional micro magnetic sensor arrays. One is a $10 \times 10$ magnetic switch array with a nickel cantilever structure, which is fabricated by electro-plating. Each cell is $1 \mathrm{~mm}$ square in size. Another one is a $10 \times 10$ Hall sensor array having same cell size. It consists of so-called a MOS-Hall element [5], which is a MOS transistor having the function of a Hall element. A Hall sensor array is simple in structure and has very high sensitivity in case of using a MOS-Hall element. Some experimental results show that the Hall sensor array is a promising magnetic sensor.

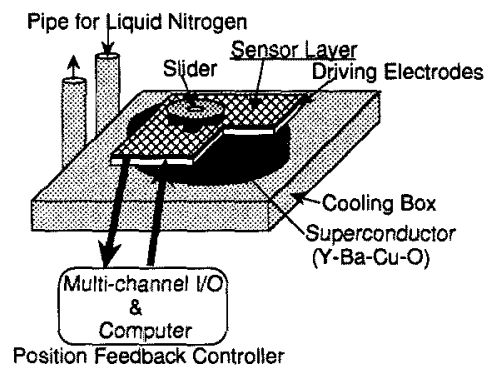

Figure 1 Micro conveyance actuator based on Superconducting Magnetic Levitation

\section{MAGNETIC SWITCH ARRAY}

When a permanent magnet gets close to a magnetic metal cantilever, the cantilever is attracted and bent as shown in Figure 2. The motion can be utilized as the mechanical magnetic switch array. The external view of the Nickel Switch Array is shown in Fig.3. One cell of the switch is $540 \mu \mathrm{m}$ square, and the cantilever beam is $20 \mu \mathrm{m}$ in width and $3 \sim 4 \mu \mathrm{m}$ in thickness. The pitch of each nickel switch is $1 \mathrm{~mm}$, and there are $10 \times 10$ switches. We have made the different stiffness of the hinge of Type A and Type B which are shown in Fig.4.

The movable electrodes and the upper fixed electrodes are fabricated by nickel electro-plating and the photo lithography process. The fabrication process is shown 
in Figure 5. First, a copper layer of $1 \mu \mathrm{m}$ in thickness is sputtered on a oxidized silicon wafer; the layer is used not only as the seeds of electro-plating, but also as the sacrificial layer for releasing. The nickel structure is fabricated by the electro-plating, after forming the negative patterns of the switch array by the photo-resist. Then, the cantilevers is released by $\mathrm{HNO}_{3}$. In parallel, the fixed electrodes are fabricated by the photo lithography process shown in Figure 5 (a) (c). A nickel switch array is finally obtained by combining the movable electrodes with the upper fixed electrodes.

When applying a large permanent magnet, which is $10 \times 10 \times 5 \mathrm{~mm}$ in size, and $440 \mathrm{mT}$ in the surface magnetic flux density, to the switch of a type A, experimental result shown in Figure 6(a) was obtained. The experimental result by applying a small magnet, which is $3 \times 2 \times 0.5 \mathrm{~mm}$ and $150 \mathrm{mT}$, to the switch of a type $B$ is shown in Figure 6(b). When applying the small magnet to a type $\mathrm{A}$, we did not get a stable result. It is evident that the type $B$ has higher sensitivity than the type A.

We observed the change in the contact resistance with respect to the magnetic flux density as shown in Figure 7. When the magnetic flux density becomes $17 m T$ by puting the permanent magnet closer, the nickel switch turns On (The contact resistances are $16 \Omega$ in Type A and $21 \Omega$ in Type B). Then the resistance decreases to about $10 \Omega$ at the magnetic flux density of $440 m T$. We can use this continuous decrease of the contact resistance to obtain more fine positioning resolution less than $1 \mathrm{~mm}$.

We estimated the contact pressure as the following the measurment and the calculation in the case of Type A. Firstly, we measured the resonance frequency by using the Piezo element and the laser displacement meter. The results of the resonance frequency are $8.7 \mathrm{kHz}$ in Type A and $7.9 \mathrm{kHz}$ in Type B. We obtain the stiffness of the hinge of Type $A$ from the following simple modeling.

$$
\begin{aligned}
& \omega=2 \pi f=\sqrt{\frac{k}{m}} \\
& m=10.6 \mu g, \quad f=8.7 k H z
\end{aligned}
$$

Here, $m$ is a mass of a Nickel plate, $f$ is a resonance frequency by the measurement of Type $\mathrm{A}$. We obtain the stiffness of $k=32[N / m]$. The gap between the Nickel structure and the upper electrode is $x_{0}=15 \mu \mathrm{m}$. We know the displacement becomes $15 \mu \mathrm{m}$, when the magnetic flux density is $B_{0}=17 \mathrm{mT}$ from Figure 7. We assume the force acting in the Nickel switch is proportional to the magnetic density. Therefore we can esti- mate the contact force, $F$, at the magnetic flux density of $B=440 \mathrm{mT}$, when the magnet attach to the glass with the upper electrodes. The contact force $F$ is calculated as follows:

$$
F=k x_{0} \frac{B-B_{0}}{B_{0}}
$$

We obtain the contact force of $F=12 m N$, by substituting $k=32[\mathrm{~N} / \mathrm{m}], x_{0}=15 \mu m, B_{0}=17 \mathrm{mT}$ and $B=440 \mathrm{~m} T$.

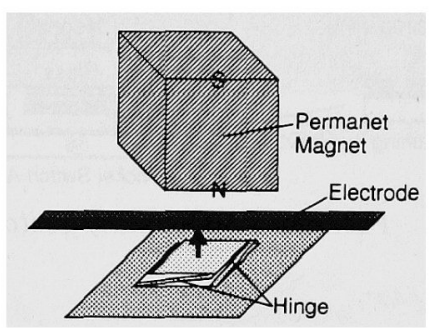

Figure 2 Principle of Nickel Switch Array
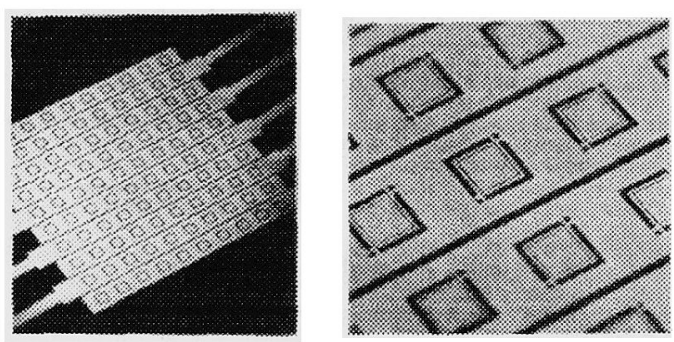

Figure 3 External view of Nickel Switch Array

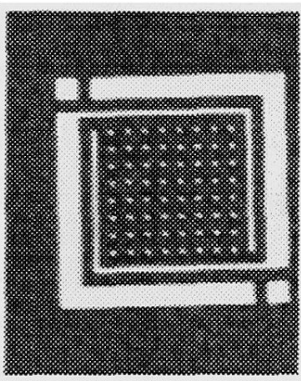

Type A

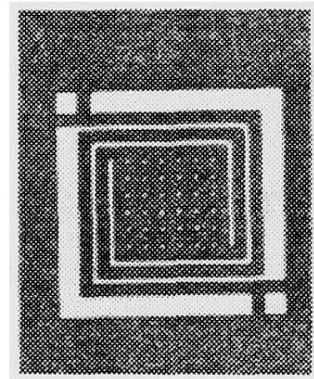

Type B
Figure 4 Hinge designs of Nickel Switch 


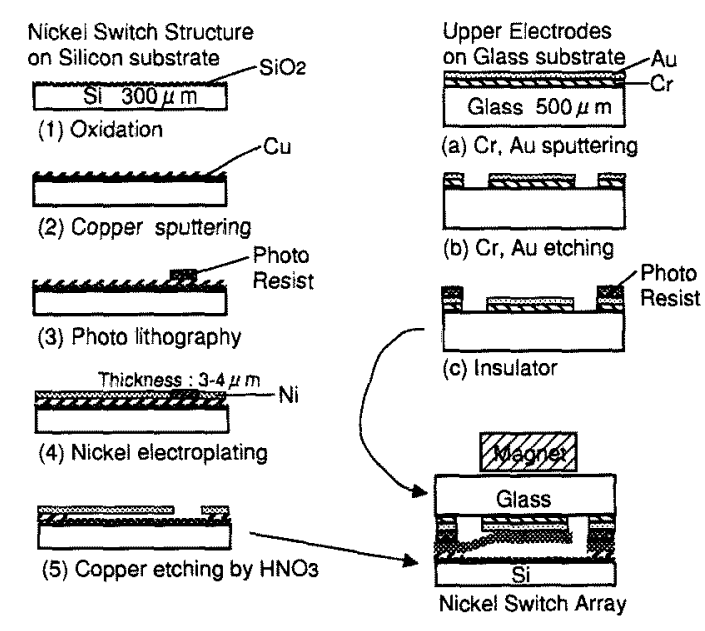

Figure 5 Fabrication process of switch array

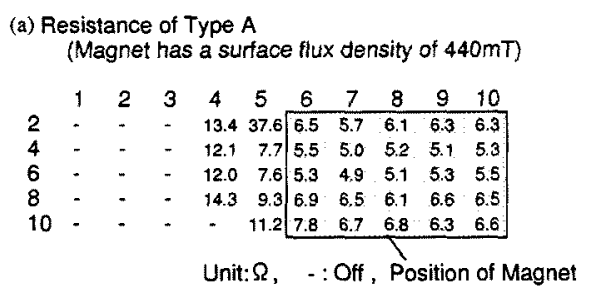

(b) Resistance of Type B

(Magnet has a surface flux density of $150 \mathrm{mT}$ )

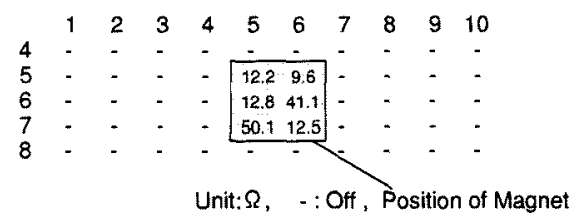

Figure 6 Contact resistance of Nickel Switch Array

\section{HALL SENSOR ARRAY}

We have developed the Hall sensor array. The principle is shown in Figure 8. To realize a two-dimensional Hall sensor array, we must avoid the interference between hall elements, because conventional Hall element (bipolar device) has finite resistance between each two terminals of its four terminals as shown in Figure 9. However, if we replace it with MOS-Hall element as shown in Figure 10, which takes ON (finite resistance) or OFF (infinite resistance) depending on the gate voltage, it is possible to avoid the interference between Hall elements. In fact, MOS-Hall element has both the function of a MOS transistor and the function of a Hall element. It is a unipolar device, reported by R.C.Gallagher in 1966, and by M.Suzuki in 1981. It is said MOS-Hall

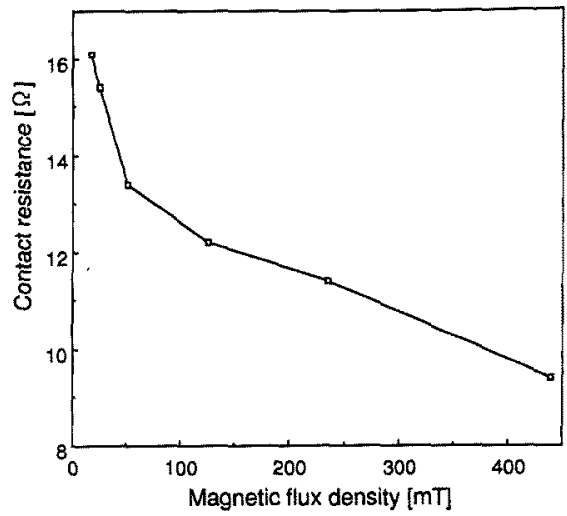

Figure 7 Contact resistance under the magnetic flux density of Type $A$

element has a very high sensitivity. When only one sensor cell in Figure 9 is selected to $O N$ after replacing conventional Hall element with MOS-Hall element, the other cells stay OFF, that is infinite resistance theoretically. Hence, a Hall sensor array needs only one pair of output terminals, to which all outputs are connected in parallel. In other words, the sensor is low cost for the latter processing circuit. Authors here propose three types of sensor cells shown in Figure 10, each of which consists of both MOS-Hall element and control circuits. Comparing these cells, we found that they have similar function, but the device of Figure 10(a) consumes very large power, because the control circuits of almost all cells pass through current at the same time. The one in Figure 10(b) passes through current only in a column. On the other hand, the one in Figure 10(c) not only consumes little power in one cell, but also has twice as large output as others.

Authors here developed a $10 \times 10 \mathrm{Hall}$ sensor array with the function of MOS-Hall element based on SOI (silicon on insulator) wafer, the cell of which is same as Figure 10(b). The size of a cell is $1 \mathrm{~mm}$ square, and MOS-Hall element is $700 \times 400 \mu m$ in size as shown in Figure 11. The fabrication process is shown in Figure 12. A dose of Boron $2.5 \times 10^{12}$ ions $/ \mathrm{cm}^{2}$ is implanted to obtain the MOS-Hall element and a dose of Phosphors $2 \times 10^{15}$ ions $/ \mathrm{cm}^{2}$ to obtain a ohmic contact. The gate $\mathrm{SiO}_{2}$ is around $100 \mathrm{~nm}$ in thickness.

Figure 13 shows the experimental result of MOS-Hall element as a MOS transistor. It shows a typical MOS characteristic and has $1.7 \mathrm{~V}$ as the threshold voltage.

When applying a magnet $(440 m T)$ to the fabricated Hall sensor array, the experimental result shown in Figure 14 was obtained. In the table, $X=1$ means the 


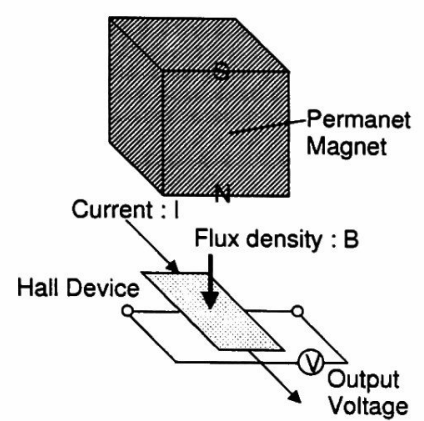

Figure 8 Principle of Hall Device Array

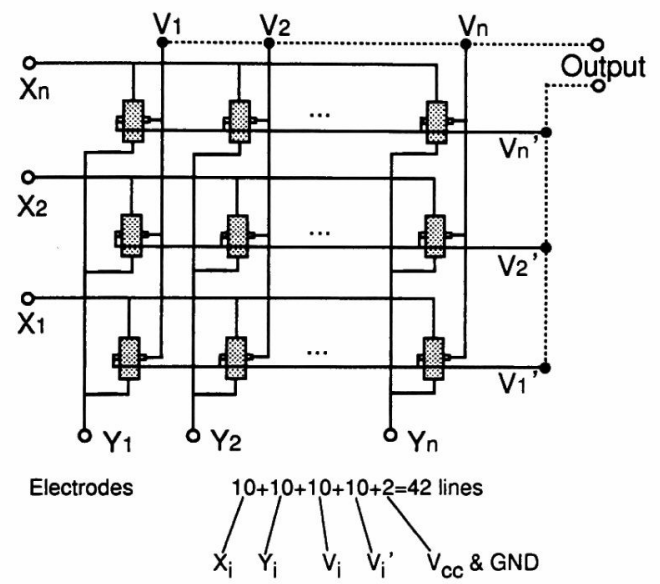

Figure 9 Electrodes connection of Hall Device Array

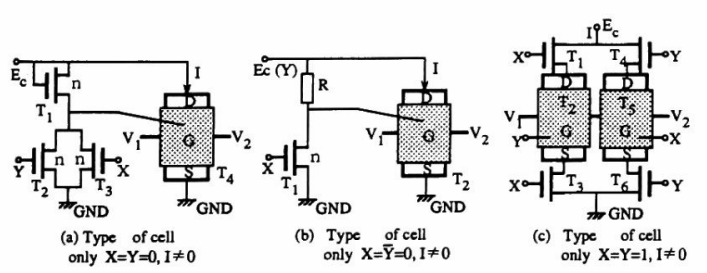

Figure 10 MOS transistor and MOS-Hall Device

gate voltage of MOS-Hall element is $\mathrm{OV}$ (see Figure 10 (b)), and I is a current from power supply. When $\mathrm{X}$ is 1 , selected MOS-Hall element is OFF and exhibits over $108 \Omega$ between two output terminals, almost no output is hence checked out. Conversely, when $\mathrm{X}$ is 0 , selected MOS-Hall element is $\mathrm{ON}$ and generates an average $26.7 \mathrm{mV}$ output, that is $420 \mathrm{~V} / \mathrm{TA}$ in product sensitivity. This product sensitivity is much larger than the one of a conventional Hall element (about 57V/T A for silicon) [6], that is because the fabricated MOS-Hall element is similar to MOS device, and has very thin in-

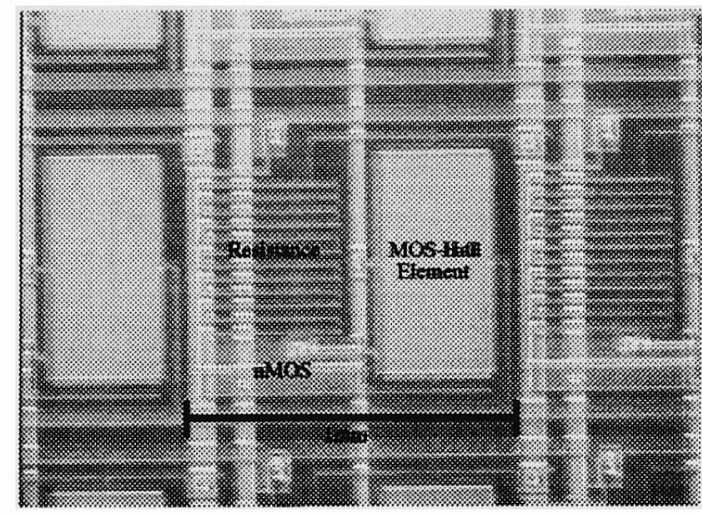

Figure 11 Close up view of Hall Device Array

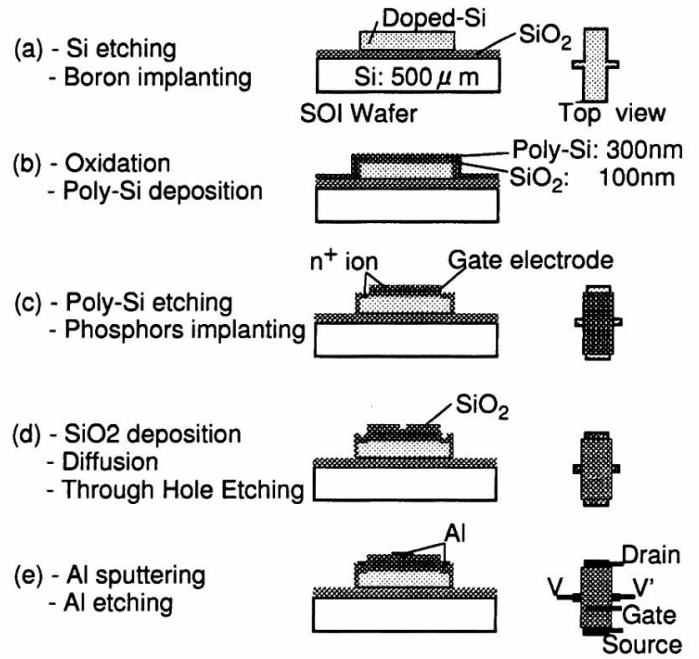

Figure 12 Fabrication process of Hall Device Array

version layer of about $10 \mathrm{~nm}$, only within which current I passes through. The high sensitivity of the device can be explained as follows: the Hall voltage is given by $k B I / d$, where $d$ is channel thickness. Because the thickness $d$ of a MOS-Hall element is very small, its sensitivity is high.

Now let us estimate the sensitivity of the device shown in Figure $10(\mathrm{c})$. When $\mathrm{I}$ is $3.5 \mathrm{~mA}$, and $E_{\mathrm{c}}$ is $10 \mathrm{mV}$, in fact, the current through a MOS-Hall element is about $0.18 \mathrm{~mA}$ according to Figure 13. Hence, we would expect $126 \mathrm{mV}$ output by using the proposed cell in Figure 10 (c), when the current $I$ is $1 m A$.

The fabricated Hall sensor array exhibits very large offset voltage, which is an obstacle to practical use without the calibration. We have to decrease it by taking some measures, for example, changing MOS-Hall element shape in the future. When gate voltage $V_{g s}$ is 


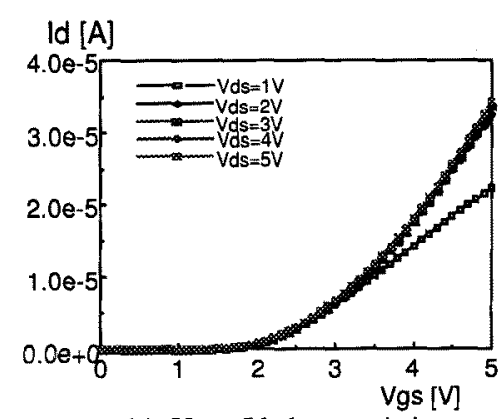

(a) Vgs - Id characteristics

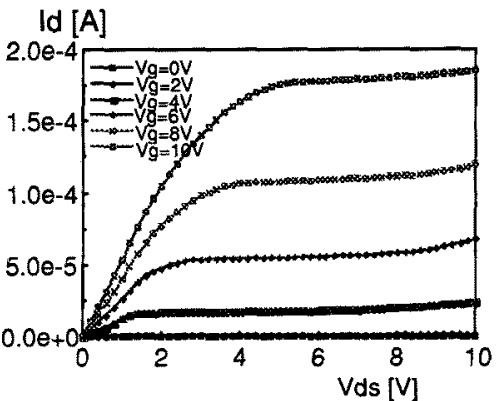

(b) Vds - Id characteristics-1

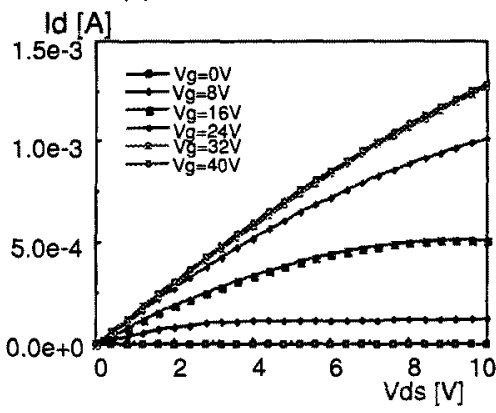

(c) Vds - Id characteristics-2

Figure 13 Characteristics of Transistor

Condition : $l=3.5 \mathrm{~mA}, Y=10 \mathrm{~V}, \mathrm{~B}=440 \mathrm{mT}$

\begin{tabular}{c|crrr}
\hline cell & $X$ & no Magnet & N-pole & S-pole \\
\hline $1-1$ & 1 & $0 \mathrm{mV}$ & $0 \mathrm{mV}$ & $0 \mathrm{mV}$ \\
$1-1$ & 0 & $311 \mathrm{mV}$ & $123 \mathrm{mV}$ & $120 \mathrm{mV}$ \\
$2-2$ & 0 & $-352 \mathrm{mV}$ & $134 \mathrm{mV}$ & $128 \mathrm{mV}$ \\
$3-3$ & 0 & $-410 \mathrm{mV}$ & $123 \mathrm{mV}$ & $132 \mathrm{mV}$ \\
\hline
\end{tabular}

Sensitivity $=420 \mathrm{~V} / \mathrm{TA}$.

Figure 14 Characteristics of Hall Device Array

equal to drive voltage $V_{d s}$, MOS-Hall element is working on saturation region according to Figure 13, and hence inversion layer is wedge-shaped. However, if $V_{g s}$ is much lager than $V_{d s}$ as shown in Figure 13. MOS-Hall element is working on linear region, and inversion layer is approximately parallel, just like a conventional Hall element. It is evident that $V_{g s} \gg V_{d s}$ is a better working condition than $V_{g s}=V_{d s}$. We are planning to realize the condition by using the circuit shown in Figure 13(c).

\section{CONCLUSIONS}

This paper proposed two types of two dimensional micro magnetic sensor arrays. One has a mechanical cantilever structure based on nickel electro-plating and fabrication process is simple. Hence, it is easy to realize a large area and low cost sensor. We intend to miniaturize its cell size furthermore for obtaining higher resolution. Another one is a Hall sensor array based on MOS process. It has a high sensitivity of $420 \mathrm{~V} / \mathrm{TA}$. We reduced the number of the electrical wire by introducing MOS switch. Authors intend to use these two types of sensors to our micro superconducting magnetic conveyance system with feedback control.

\section{ACKNOWLEDGEMENTS}

The authors are grateful to members of Fujita laboratory and Hiramoto laboratory for their advise and help in the fabrication processes.

(Manuscript received May 7,1998 )

\section{References}

(1) M.Ataka, A.Omodaka, N.Takeshima and H.Fujita: Fabrication and Operation of Polyimide Bimorph Actuators for A Ciliary Motion System, Journal of Microelectro-mechanical System, Vol.2, No.4, pp.146$150,(1993)$

(2) Y.Mita, S.Konishi and H.Fujita: Two Dimensional Micro Conveyance System with Through Holes for Electrical and Fluidic Interconnection, 1997 International Conference on Solid-State Sensors and Actuators (Transducers 97), IEEE Electron Devices Society, in Chicago, USA, Vol.1, pp.37-40, June 16-19 (1997)

(3) Haruo Nakazawa, Yasumasa Watanabe, Osamu Morita, Masaharu Edo and Eiichi Yonezawa: The Two-dimensional Micro Conveyer, 1997 International Conference on Solid-State Sensors and Actuators (Transducers 97), IEEE Electron Devices Society, in Chicago, USA, Vol.1, pp.33-36, June 16-19 (1997)

(4) Tetsuhiko Iizuka and Hiroyuki Fujita: Precise Positioning of a Micro Conveyor based on Superconducting Magnetic Levitation, IEEE, 1997 International Symposium on Micromechatronics and Human Science (MHS'97), in Nagoya, pp.131-135, October 5-8 (1997)

(5) K. Takahashi and A. Sasaki: Advanced Sensor Handbook, Published by Baifukan, p.267, (1994), in Japanese. 
(6) S. Kawahito, S. O. Choi, M. Ishida and T. Nakamura: MOS Hall Element with Three-Dimensional Microstructure, The 7th International conference on Solid-State Sensors and Actuators, Transducers 93, June 7-10, Pacifico Yokohama, Japan, pp.892-895, (1993)

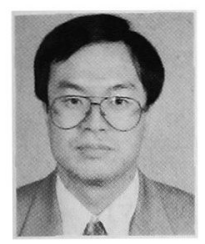

Desheng LI (Member) He received his B.E. degree from Hunan University, China, in 1982 and M.E. degree from Harbin Institute of Technology (HIT), China,in 1988 and $\mathrm{Ph} . \mathrm{D}$. degree from Saga University in 1966. He is currently a professor of HIT. His main research interests are sensor engineering, MEMS and mobile robot.

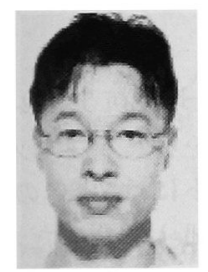

Kyoseok CHUN (Member) He received the B.S. degree in 1993, in electronic engineering from Kyung-pook national university, Taegu, Korea and the M.S. degree in electrical and electronic engineering from Toyohashi university of Technology, Toyohashi, Japan, in 1996. He is currently Ph.D student in electrical engineering of the university of Tokyo, Japan, from 1996. His research interests are bio-applications of micromachining technology.

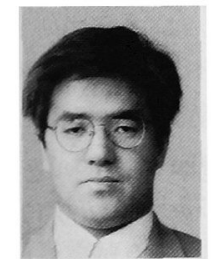

Tetsuhiko IIZUKA (Member) He received his B.S. degree in 1991 and his M.E. degree in 1993 from Waseda University. He is a technical associate in Institute of Industrial Science, the University of Tokyo. His research interests are micro mechatronics and motion control.

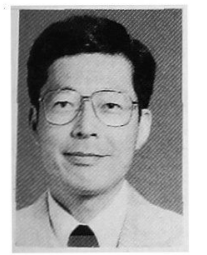

Hiroyuki FUJITA (Member) He received the B.S. degree in 1975, the M.S. degree in 1977 and the Ph.D degree in 1980, all in electrical engineering, from the University of Tokyo, Tokyo, Japan. He is currently a professor in the Institute of Industrial Science at the University of Tokyo, where he joined the faculty in 1980. His current research interests are system design and fabrication technologies of microelectromechanical systems. He also investigates autonomous distributed systems that mimic living organisms. 